\title{
Bose and Fermi gases in the early universe with self-gravitational effect
}

\author{
Yuezhen Niu and Junwu Huang \\ School of Physics and State Key Laboratory of Nuclear Physics and Technology, \\ Peking University, Beijing 100871, China \\ Bo-Qiang Ma* \\ School of Physics and State Key Laboratory of Nuclear Physics and Technology, \\ Peking University, Beijing 100871, China \\ Center for High Energy Physics, Peking University, Beijing 100871, China and \\ Center for History and Philosophy of Science, Peking University, Beijing 100871, China
}

\begin{abstract}
We study the self-gravitational effect on the equation of state (EoS) of Bose and Fermi gases in thermal equilibrium at the end of reheating, the period after quark-hadron transition and before Big Bang Nucleosynthesis (BBN). After introducing new grand canonical partition functions based on the work of Uhlenbeck and Gropper, we notice some interesting features of the newly developed EoSs with distinct behaviors of relativistic and non-relativistic gases under self-gravity. The usual negligence of the self-gravitational effect when solving the background expansion of the early universe is justified with numerical results, showing the magnitude of the self-gravitational modification of the state constant to be less than $O\left(10^{-78}\right)$. This helps us to clarify the background thermal evolution of the primordial patch. Such clarification is crucial in testing gravity theories, evaluating inflation models and determining element abundances in BBN.
\end{abstract}

PACS numbers: 98.80.-k, 04.40.-b, 05.30.-d

\section{INTRODUCTION}

In most history of our universe, gravity has been the dominant force in determining cosmological evolutions. Due to the onset of gravitational instability, primordial fluctuations generated in cosmic inflation start growing by attracting dark matter and gases nearby after the matter radiation equilibrium at a temperature approximately $4000 \mathrm{~K}$ [1]. Consequently, structures of the first generation galaxies are seeded. Shortly after the forming of proto-galaxies, the hydrogen and helium gases within them begin to condensate and make the first stars. In the formation of any astrophysical body, self-gravity is beyond question one of the key factors in determining the specific destinies of stars, quasars, black holes, and neutron stars. For example, the self-gravity of neutron stars is considered using the affine model [2, 3], where the effective relativistic self-gravitational potential is approximated with Tolman-Oppenheimer-Volkoff (TOV) stellar structure equations. Indeed, gravity, though much weaker than the other three fundamental forces, i.e., strong, weak and electromagnetic forces, with its long range of action and omnipresent character from equivalence principle of general relativity, determines most astronomical evolutions. One persuasive example showing the significance of self-gravity is the boson star. Though massive boson stars are prevented from the Heisenberg uncertainty principle, they can actually be formed in the early universe when the self-interaction of scalar particles is considered [4, 5]. Various methods are sub-

*Electronic address: mabq@pku.edu.cn sequently developed to study different features of boson stars. People deal with a system of self-gravitating bosons either by solving the Schrödinger and Poisson equations in a Newtonian way [6], or by fully accounting the relativistic effect using Klein-Gordon and Einstein equations [7]. When the Einstein gravity and the Klein-Gordon field couple with each other, exotic bosons which possess a small mass may undergo Bose-Einstein condensation driven by gravity and collapse to form boson stars in the early universe or latter in galaxy center as a candidate for dark matter $8-10]$.

If self-gravity can trigger phenomena at the early universe so dramatic like boson stars or primordial black holes [11, 12] not mentioned before, the self-gravitational effect in the primordial patch of the early universe should not be easily discarded without a robust proof. An exact evaluation of the self-gravity of relativistic particles at the extremely high energy scale before Big Bang Nucleosynthesis (BBN) (not high enough to reach the boson star critical temperature) becomes unavoidable. However, most discussions of boson stars are at relatively high energy scale $\sim 1 \mathrm{GeV}[13$, and at the same time entail the non-zero mass of scalar particles to be greater than around $10 \mathrm{eV}$ to preserve radial stability 14. But during the radiation dominated era before $\mathrm{BBN}$, main ingredients are massless photons, and the homogeneity of the early patch is preserved unlike that in boson stars. As a result, it is difficult to fully perceive the selfgravitational behavior of photons in the early universe by simply extending the methods developed for boson stars. Although there are several attempts to include the self-gravitational effect of non-relativistic gases at equilibrium, such as Monte Carlo simulations, analytic mean field methods and low density expansions 15], no method 
so far exists to directly solve the self-gravity of massless particles, i.e., photons. As a result, we try to develop an appropriate way to deal with the self-gravity of massless bosons in the early universe. The newly developed method will be applied to massive particles as well.

Before BBN, the universe can be well described as in thermal equilibrium with very high temperature and frequent interactions. Remarkable features of the early universe, homogeneity, isotropy and thermal equilibrium together can help us simplify the discussion to a great extent. Since the Equation of State (EoS) can serve as a good description of a homogeneous systems, we manage to delve into the problem with statistical physics. As is well known, once including self-interactions between particles, the corresponding partition function shall be changed. Such change further leads to modifications in the EoS. We try to uncover the self-gravitational effect by examining the corresponding statistical properties of Bose and Fermi gases first. Whether corrections from self-gravity are negligible or bear some new features not considered before shall be tested in detail in this work.

In the radiation-dominated epoch, gravities between relativistic particles are commonly neglected in existing models. When the temperature of the universe decreases to approximately $10^{11} \mathrm{~K}$, particles are in thermal equilibrium without strong nuclear interactions and the principal ingredients at that time are photons, neutrinos, electrons and their anti-particles. With the energy of the universe dominated by photons, other ingredients, whether massive particles like electrons and positrons or the massless ones, all behave radiation-like due to their frequent collisions with photons and each other at the ultra-high temperature. The matter soup at that time is suggested to follow the equation of state $P=\rho / 3$ without considering gravitational attractions between particles. From the equivalence principle, massless particles with both high kinetic energies and particle densities may possess a considerable gravitational effect. We deal with self-gravitational interactions between these thermalized particles in a Newtonian way. With a statistical approach, we can avoid the theoretical difficulties in solving equations of general relativity as well as numerical errors in actual realization, and at the same time, perceive macro-behaviors without any impediment from micro-complexity. In Sec. III we deduce a new form of grand canonical function for Bose and Fermi gases in the relativistic and the non-relativistic limits. In Sec. III, we apply the newly-developed partition function to the early universe before BBN. We also analyze the time dependent term of the EoS and set a strict upper bound on the modifications of the state constant from self-gravity. Possible influences on relating topics are discussed in Sec.IV.

\section{GRAND CANONICAL PARTITION FUNCTION FOR SELF-GRAVITATIONAL BOSE AND FERMI GASES}

In standard statistical physics, people deal with interactions between gases by including $e^{-\phi_{i j} / k T}$ in the grand canonical partition function, where $\phi_{i j}$ is the potential energy resulting from the interactions between two particles $i, j$. The potential energies $\phi_{i j}$ are set to be zero in general. Such approximation works pretty well when interaction is negligible, that is, the density-temperature ratio is extremely low. On the other hand, if the density is relatively high like that in the early universe, the factor from the self-gravitational effect of bosons and fermions becomes appreciable. As Uhlenbeck and Gropper [16] proved, the partition function of non-ideal Bose or Fermi gases should be modified as

$$
e^{-\phi_{i j} / k T}\left(1 \pm \exp \left[-4 \pi m k_{B} T r_{i j}^{2} / h^{2}\right]\right),
$$

where the plus sign is for Fermi gas.

The grand canonical partition function becomes

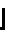




$$
\int \prod_{i j}^{i<j} e^{-\frac{G m^{2}}{r_{i j} B^{T}}}\left(1 \pm \exp \left[-4 \pi m k_{B} T r_{i j}^{2} / h^{2}\right]\right) \mathrm{d} \mathbf{r}_{i j}=\int e^{-\sum_{i<j} \frac{G m^{2}}{r_{i j} k^{T}}}\left(1 \pm \sum_{i j} \exp \left[-4 \pi m k_{B} T r_{i j}^{2} / h^{2}\right]\right) \mathrm{d} \mathbf{r}_{i j} .
$$

With $\exp \left[-4 \pi m k_{B} T r_{i j}^{2} / h^{2}\right] \ll 1$, the omissions made in Eq. (4) are justified. At the same time, we use mean field theory to include only the average effect of $r_{i j}$. The thermal equilibrium used in mean field theory is guaranteed in the early universe. As $N \rightarrow \infty$ we approximate $N(N-1) / 2 \rightarrow N^{2} / 2$, following which Eq. (4) becomes
This reveals that the upper bound of the integration corresponds to the spacial scale of the system.

The second term of Eq. (5) is

$$
\begin{aligned}
& \int e^{-\frac{N^{2} G m^{2}}{2 r_{*} B_{B} T}}\left(1 \pm \frac{N^{2}}{2} \exp \left[-4 \pi m k_{B} T r_{*}^{2} / h^{2}\right]\right) \mathrm{d} \mathbf{r}_{*} \mathrm{~d} \mathbf{r} \\
= & V^{N-1}\left\{\int e^{-\frac{N^{2} G m^{2}}{2 r_{*} k_{B} T}} \mathrm{~d} \mathbf{r}_{*} \pm \int \frac{N^{2}}{2} \exp \left[-4 \pi m k_{B} T r_{*}^{2} / h^{2}\right] \mathrm{d} \mathbf{r}_{*}\right. \\
& \left. \pm \int \frac{N^{2}}{2}\left(e^{-\frac{N^{2} G m^{2}}{2 r_{*} k_{B} T}}-1\right) \exp \left[-4 \pi m k_{B} T r_{*}^{2} / h^{2}\right] \mathrm{d} \mathbf{r}_{*}\right\},
\end{aligned}
$$

$$
\begin{aligned}
& \int \frac{N^{2}}{2} \exp \left[-4 \pi m k_{B} T r_{*}^{2} / h^{2}\right] \mathrm{d} \mathbf{r}_{*} \\
= & 4 \pi \int_{0}^{r_{0}} \frac{N^{2}}{2} \exp \left[-4 \pi m k_{B} T r_{*}^{2} / h^{2}\right] r_{*}^{2} \mathrm{~d} r_{*} \\
= & 4 \pi \int_{0}^{\infty} \frac{N^{2}}{2} \exp \left[-4 \pi m k_{B} T r_{*}^{2} / h^{2}\right] r_{*}^{2} \mathrm{~d} r_{*} \\
= & 2^{-5 / 2} \pi^{3 / 2} N^{2} \Lambda_{\text {nonrela }}^{3}
\end{aligned}
$$

ment and the second term corresponds to usual BoseEinstein or Fermi-Dirac statistics, while the last term is a newly-introduced coupling term from the gravity of bosons or fermions. Again, the upper sign is for Fermi gas.

In the early universe, particle number and energy density are relatively high with temperature $\sim 10^{11} \mathrm{~K}$, i.e., $\exp \left[-N^{2} G m^{2} / 2 r_{*} k_{B} T\right] \sim 1$. To integrate the first term of Eq. (5), we expand it into the the first-order Taylor series and get

$$
\begin{aligned}
\int e^{-\frac{N^{2} G m^{2}}{2 r_{*} B_{B}^{T}}} \mathrm{~d} \mathbf{r}_{*} & =4 \pi \int_{0}^{r_{0}}\left(1-\frac{N^{2} G m^{2}}{2 r_{*} k_{B} T}\right) r_{*}^{2} \mathrm{~d} r_{*} \\
& =V-\left(\frac{9 \pi}{16}\right)^{1 / 3} V^{2 / 3} \frac{N^{2} G m^{2}}{k_{B} T} .
\end{aligned}
$$

where $\Lambda_{\text {nonrela }}=h / \sqrt{2 \pi m k_{B} T}$ is the thermal de Broglie wavelength, which should be much smaller than the radius of our interested system. If the scale of the system is equal to or smaller than the thermal de Broglie wavelength, a situation which might happen at a much earlier time, such approximation may not be valid, and we should use the error function $\operatorname{erf}(x)$ in the integration instead. Again applying the first-order Taylor expansion, we get the remaining term

$$
-\frac{N^{4} \pi G m^{2}}{k_{B} T} \int_{0}^{r_{0}} \exp \left[-4 \pi m k_{B} T r_{*}^{2} / h^{2}\right] r_{*} \mathrm{~d} r_{*}=-\frac{N^{4} \pi G m^{2}}{4 k_{B} T} \Lambda_{\text {nonrela }}^{2}
$$

Finally, we find the new grand canonical partition function

$$
\Xi=\left\{1-\frac{G m^{2}}{k_{B} T}\left(\frac{9 \pi}{16}\right)^{1 / 3} V^{-1 / 3} \pm \frac{\pi^{3 / 2} \Lambda_{\text {nonrela }}^{3}}{2^{5 / 2} V} \frac{\partial^{2}}{\partial \alpha^{2}} \mp \frac{\pi G m^{2}}{4 k_{B} T V} \Lambda_{\text {nonrela }}^{2} \frac{\partial^{4}}{\partial \alpha^{4}}\right\} \exp \left[e^{-\alpha} \frac{V}{\Lambda_{\text {nonrela }}^{3}}\right]
$$


with which we can obtain the total energy by $E=$ $\partial \ln \Xi / \partial \beta$ with $\beta=1 / k_{B} T$, and then the entropy. As suggested above, this partition function is applicable when the scale of the system is much larger than the thermal de Broglie wavelength. However, such premises may be wavered at a much earlier stage. The first term of the partition function is the grand canonical function for ideal Boltzmann gas. The second term represents the self-gravitational correction of Boltzmann gas, and its magnitude corresponds to the portion that gravitational energy $E_{p} \sim G m^{2} / V^{1 / 3}$ contributes to the total energy $E_{\text {total }} \sim k_{B} T$. In turn it manifests whether gravitational correction is important in dealing with the evolution of the universe. The third term is the correction from identical principle, and its magnitude (proportional to the cubic square of thermal de Broglie wavelength over the volume of the system $V$ ) evaluates how strong quantum identity is. As a whole, the self-gravitational effect on fermions is restricted by both the strength of gravity and the intensity of quantum effect.

\section{B. The relativistic case}

For highly relativistic particles, the rest mass is negligible compared with the kinetic energy, thus the dispersion relation becomes $E_{k}=p c$. Then the grand canonical partition function is found to be

$$
\Xi=\sum_{N} \frac{e^{-\alpha N}}{N ! h^{3 N}} \int e^{-\sum_{i} \frac{p_{i} c}{k_{B} T}} \mathrm{~d} \mathbf{p}_{i} \int e^{-\sum_{i<j} \frac{G p_{i} p_{j}}{r_{i j} k_{B} T c^{2}}}\left(1 \pm \sum_{i<j} \exp \left[-4 \pi^{2} p_{i} k_{B} T r_{i j}^{2} / h^{2}\right]\right) \mathrm{d} \mathbf{r}_{i j}
$$

We can solve it exactly by diagonalizing $\sum_{i<j} \frac{G p_{i} p_{j}}{r_{i j} k_{B} T c^{2}}$ first. But such treatment is proved to be unnecessary since, on one hand, the self-gravitational effect in the relativistic case acts as a correction term, and on the other hand, for our problem in the homogeneous and isotropic Robertson-Walker metric background at exceptionally high temperature, relativistic particles are in a thermal equilibrium state evolving in a radiation manner resulting from frequent collisions. Therefore, we expect self-gravity alone will not significantly destroy this homogeneity directly shown by CMB observations. All particles in the patch then possess the same characteristic average momentum due to the frequent collision. We then approximate the summation as

$$
-\sum_{i} \frac{p_{i} c}{k_{B} T}-\sum_{i<j} \frac{G p_{i} p_{j}}{r_{i j} c^{2} k_{B} T} \approx \sum_{i}\left(\frac{c}{k_{B} T}-\sum_{j} \frac{G \bar{p}}{r_{i j} c^{2} k_{B} T}\right) p_{i}
$$

where $\bar{p}$ is the average momentum of the particles in the system. So the grand canonical partition function becomes

$$
\begin{aligned}
\Xi= & \sum_{N} \frac{e^{-\alpha N}}{N ! h^{3 N}}\left\{\prod_{i} \int e^{-\left(\frac{c}{k_{B} T}+\sum_{j} \frac{G \bar{p}}{2 r_{i j} c^{2} k_{B} T}\right) p_{i}} \mathrm{~d} \mathbf{p}_{i} \mathrm{~d} \mathbf{r}_{i}\right. \\
& \left. \pm \prod_{i} \int e^{-\left(\frac{c}{k_{B} T}+\sum_{j} \frac{G \bar{p}}{2 r_{i j} c^{2} k_{B} T}+\sum_{j} 4 \pi^{2} \frac{k_{B} T r_{i j}^{2}}{h^{2} c^{2}}\right) p_{i}} \mathrm{~d} \mathbf{p}_{i} \mathrm{~d} \mathbf{r}_{i}\right\} \\
= & \sum_{N} \frac{e^{-\alpha N}}{N ! h^{3 N}} \int \frac{(8 \pi)^{N}}{\left(\frac{c}{k_{B} T}+\sum_{j} \frac{G \bar{p}}{2 r_{i j} c^{2} k_{B} T}\right)^{3 N}} \mathrm{~d} \mathbf{r}_{i} \\
& \pm \sum_{N} \frac{e^{-\alpha N}}{N ! h^{3 N}} \int \frac{(8 \pi)^{N}}{\left(\frac{c}{k_{B} T}+\sum_{j} \frac{G \bar{p}}{2 r_{i j} c^{2} k_{B} T}+\sum_{j} 4 \pi^{2} \frac{k_{B} T r_{i j}^{2}}{h^{2} c^{2}}\right)^{3 N}} \mathrm{~d} \mathbf{r}_{i} .
\end{aligned}
$$

To integrate the first term in Eq. (11), we perform the Taylor expansion to the first order knowing the magni- tude of gravity being small outside the critical sphere with radius $r_{m}$ 


$$
\int_{r_{m}}^{r_{0}} \frac{(8 \pi)^{N}}{\left(\frac{c}{k_{B} T}+\sum_{j} \frac{G \bar{p}}{2 r_{i j} c^{2} k_{B} T}\right)^{3 N}} \mathrm{~d} \mathbf{r}_{i}=\int \frac{(8 \pi)^{N}}{\left(\frac{c}{k_{B} T}\right)^{3 N}}\left(1-3 N \sum_{j} \frac{G \bar{p}}{2 r_{i j} c^{3}}\right) \mathrm{d} \mathbf{r}_{i}
$$

The integration from 0 to $r_{m}=G \bar{p} / c^{3}$

$$
\int_{0}^{r_{m}} \frac{(8 \pi)^{N}}{\left(\frac{c}{k_{B} T}+\sum_{j} \frac{G \bar{p}}{2 r_{i j} c^{2} k_{B} T}\right)^{3 N}} \mathrm{~d} \mathbf{r}_{i}
$$

is neglected since its magnitude is proportional to $r_{m}^{3 N+1}$, much smaller than the first one in our analysis when gravity contributes as a correction term.

Under the same mean field approximation, Eq. (12) becomes

$$
\frac{(8 \pi)^{N}}{\left(\frac{c}{k_{B} T}\right)^{3 N}}\left(V-\left(\frac{\pi}{6}\right)^{1 / 3} \frac{9 \pi G N^{2} \bar{p}}{c^{3}} V^{2 / 3}\right) V^{N-1}
$$

Before going on to integrate the second term of Eq. (11), we first compare the magnitude of different terms in the denominator $\frac{c}{k_{B} T}+\sum_{j} \frac{G \bar{p}}{2 r_{i j} c^{2} k_{B} T}+$ $\sum_{j} 4 \pi^{2} \frac{k_{B} T r_{i j}^{2}}{h^{2} c^{2}}$. When $\frac{c}{k_{B} T} \backsim 4 \pi^{2} \frac{k_{B} T r_{i j}^{2}}{h^{2} c^{2}}$, i.e. $r_{i j} \backsim \frac{h c^{3 / 2}}{k_{B} T}$, the corresponding energy density can be approximated as $U \backsim \frac{h c}{r_{i j}^{4}} \backsim 10^{86}$ in the Planck unit. However, the actual energy density is of magnitude $10^{22}$ [17], meaning that the first term is $O\left(10^{-16}\right)$ compared with $\pi^{2} \frac{k_{B} T r_{i j}^{2}}{h^{2} c^{2}}$. Besides, since in our interested time period, the temperature varies within one order in magnitude, so long as the number density does not change beyond $O\left(10^{21}\right)$, i.e. the volume of the universe increases by less than $O\left(10^{21}\right)$ within 1s (such circumstances are fully guaranteed in a classical radiation-dominated universe), $\frac{c}{k_{B} T}$ can then be safely neglected. But for some earlier stages this treatment may fail. In the same way, $\frac{G \bar{p}}{r_{i j} c^{2} k_{B} T}$ is $O\left(10^{-48}\right)$ compared with the third term and can be justifiably omitted if the number density varies within $O\left(10^{40}\right)$. Neglecting the first two terms after careful magnitude evaluation, we have

$$
\int \frac{(8 \pi)^{N}}{\left(\frac{c}{k_{B} T}+\sum_{j} \frac{G \bar{p}}{r_{i j} c^{2} k_{B} T}+\sum_{j} 4 \pi^{2} \frac{k_{B} T r_{i j}^{2}}{h^{2} c^{2}}\right)^{3 N}} \mathrm{~d} \mathbf{r}_{i} \ll \frac{(8 \pi)^{N}}{\left(\frac{c}{k_{B} T}\right)^{3 N}}\left(V-\left(\frac{\pi}{6}\right)^{1 / 3} \frac{9 \pi G N^{2} \bar{p}}{c^{3}} V^{2 / 3}\right) V^{N-1} .
$$

Therefore only the first integration contributes to our final result, corresponding to a unification of Bose and Fermi distributions under the relativistic limit. The grand canonical partition function for relativistic particles including the self-gravitational effect becomes

$$
\Xi=\left[1-\left(\frac{\pi}{6}\right)^{1 / 3} \frac{9 \pi G k_{B} T}{c^{4} V^{1 / 3}} \frac{\partial^{2}}{\partial \alpha^{2}}\right] \exp \left[e^{-\alpha}\left(\frac{8 \pi k_{B} T}{c h}\right)^{3} V\right]=\left[1-6^{2 / 3} \frac{3 \pi G h}{\Lambda_{\text {rela }} c^{3} V^{1 / 3}} \frac{\partial^{2}}{\partial \alpha^{2}}\right] \exp \left[e^{-\alpha} \frac{V}{\left(\Lambda_{\text {rela }}\right)^{3}}\right]
$$

where $\Lambda_{\text {rela }}=c h / 2 \pi^{1 / 3} k_{B} T$ is the thermal de Broglie wavelength for massless particles. In Eq. (16), the first term is merely the ordinary ideal Boltzmann gas term, while the second is induced by self-gravity, proportional to $G h / \Lambda_{\text {rela }} c^{3} V^{1 / 3}$, i.e. the gravitational potential energy over the self-energy of particles. By comparing the two terms we can catch a glimpse of how gravity acts on the statistical properties of the relativistic particles.

\section{RESULT IN THE EARLY UNIVERSE}

Before applying the newly developed method to a specific background, we first review the whole evolution of the early universe. In the very early stages of the evolution, to resolve several problems in the Big Bang cosmology, like the horizon problem, magnetic-monopole problem, structure formation problem and so on, the universe is suggested to undergo an inflation between time 
scales $10^{-43} \mathrm{~s}$ and $10^{-34} \mathrm{~s}$. By the end of the inflation, the background temperature is roughly $10^{28} \mathrm{~K}$ when strong, weak and electromagnetic forces are unified. As the temperature cools down, the strong force freezes out, weak and electromagnetic interactions are subsequently off stage and before $10^{-4} \mathrm{~s}$. Afterward, neutrinos decouple at $T \sim 10^{9} \mathrm{~K}$. Then Big Bang Nucleosynthesis begins at temperature $10^{9} \mathrm{~K} \rightarrow 10^{3} \mathrm{~K}$. At the end of BBN, photons decouple from matter, and form the cosmic microwave background radiation we observe today. To use our statistical method in studying the self-gravitational effect, assumptions of homogeneity, isotropy and thermal equilibrium should be justified. We thus choose to study the universe before BBN when three criteria are largely met and the background temperature $T_{\mathrm{th}}$ is of magnitude $10^{11} \mathrm{~K}$ [17], well below the corresponding thermal energy of the rest mass of $\pi$ mesons and strong interactions can be neglected. Matter and radiation in the universe at that time are in thermal equilibrium, resulting from the rapid collisions in the exceedingly high temperature despite of the fast universal expansion. By identifying particles whose rest masses are below the corresponding thermal energy of the background temperature $T_{\mathrm{th}}$, we find the main ingredients to be electrons, neutrinos, their anti-particles and photons. These abundant genres are highly relativistic. For example, the rest energy of the electron corresponds to the thermal energy at temperature $\sim 10^{9} \mathrm{~K}$, two orders of magnitude smaller than the background temperature. And non-relativistic particles, mainly protons and neutrons, constitute only an extremely small portion of the whole ingredients, approximately $10^{-10}$ as many as that of relativistic ones. Below we deal with relativistic and non-relativistic particles separately, though the latter is unable to significantly influence the background evolution.

\section{A. Thermalized relativistic particles}

At a temperature higher than $8000 \mathrm{~K}$, the universe is radiation dominated. Consequently, relativistic particles are undoubtedly the main ingredients during our studying period with a temperature approximately $10^{11} \mathrm{~K}$. And both massive and massless components act in a radiationlike manner due to frequent collisions. We use the Planck unit $k_{B}=h=c=G=1$ in all calculations and some of our discussions below. Moreover, the volume $V_{\text {init }}$, which is about four light years in radius in our partition function, corresponds to the biggest thermalized region constrained by CMB anisotropy and grows as the extension of particle horizon of the primordial patch. Though the exact size of the early universe at this time is not determined yet. The time span of our study is $0.1 \mathrm{~s} \sim 1 \mathrm{~s}$. We consider the relativistic bosons first and later find that fermions behave similarly as expected.
Their grand canonical partition function is

$$
\Xi=\left[1-6^{2 / 3} \frac{3 \pi G h}{\Lambda_{\text {rela }} c^{3} V^{1 / 3}} \frac{\partial^{2}}{\partial \alpha^{2}}\right] \exp \left[e^{-\alpha}\left(\frac{V}{\left(\Lambda_{\text {rela }}\right)^{3}}\right)\right],
$$

The chemical potential is set to be zero since the particle number of the photon, which is the main ingredient, does not conserve. Here, instead of directly solving the Friedman equation with the density and pressure derived in the new grand canonical partition function, we approximate the temperature evolution the same as that in the classical radiation-dominated model neglecting the selfgravitational effect

$$
T=a t^{-1 / 2}
$$

where $a$ is a constant to be determined by the initial condition. Combining Eqs. (17) and (18), we numerically find that the density of relativistic particles relies on time as

$$
\rho=\frac{b_{1}}{t^{2}}+\frac{\tilde{b}_{1}}{\sqrt{t} V}
$$

where $b_{1}$ and $\tilde{b}_{1}$ are separately $6.58 * 10^{150} \mathrm{~J} \cdot \mathrm{s}^{2} / \mathrm{m}^{3}$ and $7.09 * 10^{18} \mathrm{~J} \cdot \mathrm{s}^{2}$. This differs from the conventional result without considering the self-gravitational effect where $\rho \sim T^{4} \sim 1 / t^{2}$. That is, once including the self-gravity, energy density decreases much slower. This disparity in energy density can be explained as an increase in gravitational potential caused by the rapid elongation of particle separation in cosmic expansion. This remedies the conventional energy lost with the decrease of particle density and the extension of particle wavelength. The pressure behaves as

$$
P=\frac{b_{1}}{3 t^{2}}+\frac{\tilde{b}_{2}}{\sqrt{t} V},
$$

where $\tilde{b}_{2}=1.67 * 10^{18} \mathrm{~J} \cdot \mathrm{s}^{2}$. As a result, the state constant no longer stays invariant but instead relies on time

$$
w=\frac{1}{3}\left(1-\frac{d_{1}}{\sqrt{t} V}\right)
$$

where $d_{1}$ has a numerical value $3.97 \times 10^{30}$ in Planck unit which is $9.69 \times 10^{-95} \mathrm{~s}^{1 / 2} \cdot \mathrm{m}^{3}$. Knowing that during our studying period $t \sim 10^{42}$ in Planck unit, or $0.1 \mathrm{~s}$ in standard unit, and the volume is $V \sim 10^{156}$ in Planck unit, or corresponds to four light years in radius justified by CMB observation, we find that the correction term in Eq. (21) is of the magnitude $O\left(10^{-147}\right)$. Since the universe is radiation dominated at this time, we find the correction term $d_{1} / \sqrt{t} V \sim t^{-1}$. Differing from the nonrelativistic case below, the modification from self-gravity rapidly diminishes as time goes on and volume expands.

Besides, we can roughly estimate the modification term at much earlier times, such as the end of inflation when $t \sim 10^{-34} \mathrm{~s}$ and $V \sim 10^{-51} V_{\text {init }}$, to be $O\left(10^{-79}\right)$. Thus the estimated magnitude of the modification is comparable to the effect in the non-relativistic case. 


\section{B. Thermalized non-relativistic nuclear particles}

Although massive nuclear particles contribute only a negligible portion to the whole energy makeup during the period we study, their abundances are crucial in determining the late time nuclear synthesis. As a result of the rapid expansion, the energy density of the radiation decreases swiftly as $a^{-4}$ in classical treatment, while matter density such as that of protons and neutrons decreases much slower as $a^{-3}$. Additionally, relativistic particles and their anti-particles annihilate with each other as temperature goes down, and the non-relativistic nuclear particle component, therefore, becomes increasingly important. The non-relativistic feature of nuclear particles shall preserve as temperature decreases throughout the thermalization and into nuclear synthesis. Since most of the nuclear particles are fermions, we consider only non-relativistic fermion gases without loss of generality. We can safely neglect the chemical potential of Bose gases since the photon number is not conserved. But for non-relativistic Fermi gases, at extremely high temperature, such a simplification may no longer be appropriate. Fortunately, it turns out that the chemical potential fails to show up in density and pressure expressions to the first and second order approximations in our result. This not only significantly simplifies the calculation, but also justifies the usual negligence of chemical potential in the evolution of non-relativistic fermions in the early universe [1].

For massive fermions, the grand canonical partition function is

$$
\Xi=\left\{1-\frac{G m^{2}}{k_{B} T}\left(\frac{9 \pi}{16}\right)^{1 / 3} V^{-1 / 3} \pm \frac{\pi^{3 / 2} \Lambda_{\text {nonrela }}^{3}}{2^{5 / 2} V} \frac{\partial^{2}}{\partial \alpha^{2}} \mp \frac{\pi G m^{2}}{4 k_{B} T V} \Lambda_{\text {nonrela }}^{2} \frac{\partial^{4}}{\partial \alpha^{4}}\right\} \exp \left[e^{-\alpha} \frac{V}{\Lambda_{\text {nonrela }}^{3}}\right]
$$

We then obtain the energy density in a conventional way

$$
\begin{gathered}
U=-\frac{\partial}{\partial \beta} \ln \Xi, \\
\rho=-\frac{U}{V}=\rho(\beta, V) .
\end{gathered}
$$

That is, the density is a function of $\beta$ and volume, while $\beta$ and $\mathrm{V}$ vary in time. Here the background temperature evolution can be well approximated by $T \sim t^{-1 / 2}$ during the radiation dominated period. In the time span we are studying, $\beta$ varies within one order of magnitude. To simplify the problem correspondingly, we set the temperature to be $10^{11} \mathrm{~K}$ and solve the density evolution. The mass of general non-relativistic particles in calculation is chosen to be the neutron mass, since masses of other non-relativistic nuclear particles at that time differ only by a coefficient within one order of magnitude.

The expression for $\rho$ becomes exceedingly lengthy. Facing a huge magnitude range which unavoidably exceeds the calculation limit in the computer, to evaluate the expression without any numerical reduction is impossible. However, after observing the density behavior within a small volume range, an asymptotic behavior is found. We then analyze the density behavior as volume radius increases to four light years. After careful magnitude analysis, we find that as long as the volume of the system exceeds $10^{-70} V_{\text {init }}$, where $V_{\text {init }}$ is the initial volume of the primordial patch. Such a requirement is thus safely guaranteed in the primordial patch before BBN. The first-order density behavior relying on the temperature is found to be

$$
\rho=c_{1}\left(\frac{1}{\beta}\right)^{5 / 2}=c_{1} T^{5 / 2}
$$

where $c_{1}$ equals $1.27 \times 10^{-28}$ in Planck unit, or $3.9 \times$ $10^{3} \mathrm{~J} \cdot \mathrm{K}^{5 / 2} / \mathrm{m}^{3}$. We use the extreme condition of the early universe between $0.1 \mathrm{~s}$ and $1 \mathrm{~s}$ to numerically reduce the expression and find that the result goes back to the ideal fermion case, as is shown in Fig. 1. Applying the same method, a similar asymptotic behavior of the first order can be found in the pressure-volume relation. We notice that strange negative pressure occurs in the transplanckian scale due to the failure of statistical physics. However, as the radius of the patch grows to four light years, which is the initial value of our calculation, the pressure again becomes almost volume-independent. The reduced pressure is

$$
P=c_{2}\left(\frac{1}{\beta}\right)^{5 / 2}=c_{2} T^{5 / 2}
$$

where $c_{2}=2.60 \times 10^{3} \mathrm{~J} \cdot \mathrm{K}^{\frac{5}{2}} / \mathrm{m}^{3}$, as is shown in Fig. 2.

Then the equation of state in the specific time span becomes

$$
\rho=w P
$$

where the state constant $w=1.5$ to the first-order approximation, as can be seen in Fig. 1. The recurrence back to classical case implies that the gravitational effect on non-relativistic gases is quite small and can be safely neglected to the first order. We carry on the calculation 


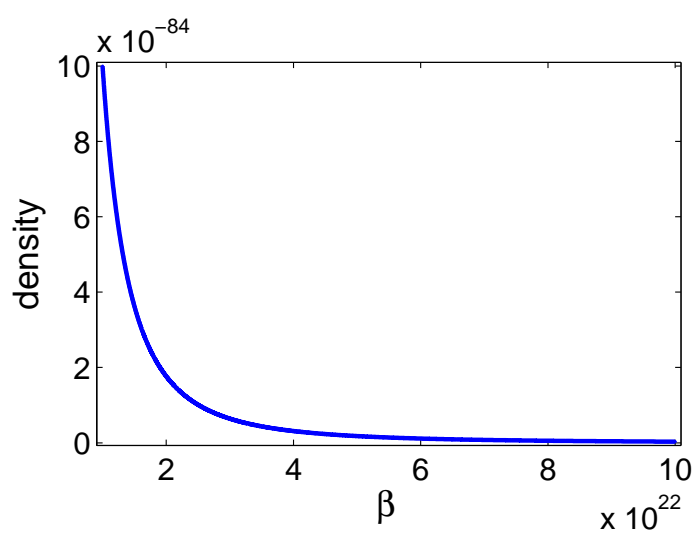

FIG. 1: The reduced density $\beta$ relation in Planck unit.

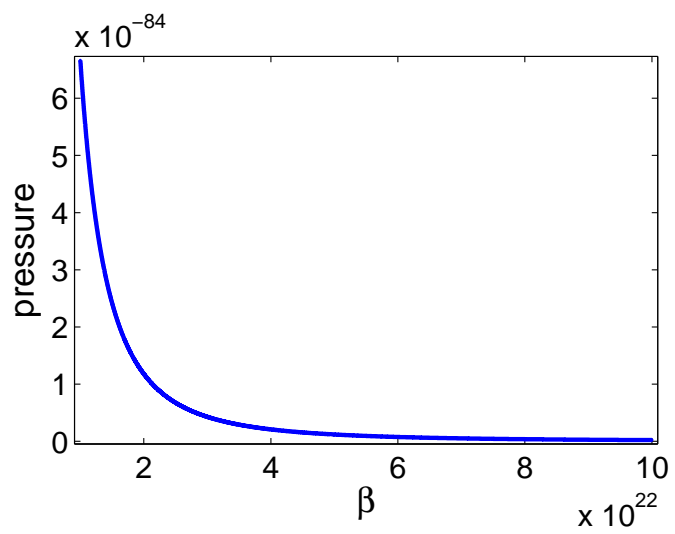

FIG. 2: The reduced pressure $\beta$ relation in Planck unit.

to the second order and find the density and the pressure behave as

$$
\begin{aligned}
& \rho=c_{1}\left(\frac{1}{\beta}\right)^{5 / 2}+\frac{\tilde{c}_{1}}{\beta}, \\
& P=c_{2}\left(\frac{1}{\beta}\right)^{5 / 2}-\frac{\tilde{c}_{2}}{\beta},
\end{aligned}
$$

where $\tilde{c}_{1}$ and $\tilde{c}_{2}$ have numerical values $1.37 *$ $10^{-70} \mathrm{~J} \cdot \mathrm{K} / \mathrm{m}^{3}$ and $2.06 * 10^{-77} \mathrm{~J} \cdot \mathrm{K} / \mathrm{m}^{3}$ respectively. The state constant to the second order evolves in time

$$
w=\frac{2}{3}\left(1-\frac{c_{3}}{T^{3 / 2}}\right),
$$

where $c_{3}$ equals $3.54 * 10^{-25} \mathrm{~K}^{3 / 2}$, with temperature $T \sim 10^{11} \mathrm{~K}$. Thus we find the total modification induced by the self-gravitational effect in the EoS to the second order acts as an additional term of the magnitude $O\left(10^{-89}\right)$ and increases over time as $c_{3} / T^{3 / 2} \sim t^{3 / 4}$. Though relatively small, we can see that such a term tends to decrease the pressure between non-relativistic particles as a manifestation of gravitational attraction. Besides, the deviation grows as temperature cools down, and finally regains its power in the matter-dominated era. The whole analysis serves as a robust test to the justification of discarding self-gravity when solving the evolution of the non-relativistic nuclear particles during the radiation-dominated period.

\section{REMARKS}

In general relativity, any kind of dynamic evolution, if inducing local energy fluctuation, cannot escape the gravitational back-reaction, i.e. the change of space time geometry itself. From the principle of minimal coupling, such dramatic feature of general relativity indeed does not affect different kinds of interactions significantly enough in most cases. Consequently, the theory of general relativity is poorly tested except in a weak-field limit within small regions like solar systems and radio pulsars, no bigger than galaxy scale. However, the Friedman equation using Robertson-Walker metric from general relativity is widely utilized in solving the homogeneous and isotropic expansion of the universe. A test of general relativity in a large scale of cosmic size then proves crucial and necessary in order to justify the foundation of modern cosmology. One of some promising methods is to use particle element abundances [18] to restrict expansion rate before $\mathrm{BBN}$.

The weak interaction before BBN between neutrons and protons should be frequent enough to counter expansion in maintaining equilibrium. Accordingly, once the expansion speeds up, the weak interaction between neutrons and protons will be out of equilibrium at higher temperature, causing a larger neutron-to-proton ratio. In turn, the abundances of deuterium, helium-3, and helium- 4 are increased. Knowing that helium- 4 is hard to destroy during galaxy formation, observations of helium4 abundance can help test the expansion rate and the cosmological constant as previous work suggested [18]. Modifications of the Friedman equation in their work can be parameterized as

$$
\left(\frac{\ddot{a}}{a}\right)=-\frac{4 \pi G}{3} \rho\left[1+\chi\left(P / 3 \rho c^{2}\right)\right]
$$

where $\chi$ corresponds to the modified state constant $w$ in our case, see Eq. (21) and Eq. (30). In existing observation results, such as those by WMAP [19], Peimbert et al. 20], and Steigman 21], the accuracy of the constraint of $\chi$ is of the magnitude $O(0.1)$, well beyond the self-gravitational effect in our result. Thus, we can exclude self-gravity as the dominant mechanism behind any observable deviation in this method.

Finally, reexamining the derived formula Eq. (21) for state constant, we compare it with the result in boson stars [8], and find that $w$ in both cases increases as the 
volume of the system expands. The merging similarities of the self-gravitational effect between two distinct circumstances are illuminating to understand the fundamental properties of gravity itself.

\section{CONCLUSION}

In this paper, we try to evaluate the self-gravitational effect in Bose and Fermi gases at the early universe and set an exact bound on the magnitude of the modification. We study the Bose and Fermi gases before BBN synthesis in thermal equilibrium at a temperature in the vicinity of $10^{11} \mathrm{~K}$, when particles collide relativistically with each other frequently enough to maintain thermalization in spite of the significant cosmic expansion. We formulate new grand canonical partition functions based on the proof Uhlenbeck and Gropper made on non-ideal Bose and Fermi gases. Taking advantage of the severe state of the early universe, we reduce the modified density and pressure expressions and derive the equations of state of relativistic and non-relativistic gases, discovering that self-gravity manifests itself in a simple way. In both non-relativistic and relativistic cases, we find the deviation from ideal ones to be small. This justifies the neglecting of the self-gravitational effect when dealing with non-relativistic particles. However, dynamical behavior appears when considering the second order effect. Our result serves as a robust proof for neglecting self-gravity when solving the evolutions of relativistic Bose and Fermi gases as well as the thermalized non-relativistic Fermi gases. Additionally, we distinguish different effects of self-gravity on the relativistic and the non-relativistic particles. In thermalized relativistic particles, discrepancies between boson and fermion statistics become unapparent, and the self-gravitational effect of magnitude $O\left(10^{-147}\right)$ decreases with time. This is naturally expected as matter becomes more and more dominant in the energy content while the energy density of radiation undergoes a more rapid dilution. Gravitational attrac- tion consequently diminishes much faster in radiation components than in matter. While in non-relativistic Fermi gases, the self-gravitational effect, though still very small of the magnitude $O\left(10^{-89}\right)$, increases over time as the temperature cooling down dramatically and finally becomes significant in the matter-dominated era when both homogeneity and isotropy break down and the EoS description is no longer appropriate. As to the evolution of the background metric, we find that self-gravity of any kind will not strongly affect the conventional result using EoS of ideal relativistic and non-relativistic boson and fermion statistics. Thus the previously not fully justified negligence of self-gravity is theoretically studied from a statistical perspective when dealing with cosmic evolution before BBN. However, our study is restricted to a given temperature range, and the same result may no longer be true at the very early stage of the universe as can be seen in Eq. (21). At a much earlier epoch, when the volume of the universe is small enough with the basic assumption of our statistical approach of thermal equilibrium still satisfied, self-gravity may no longer be negligible from our result. Thus, further study should be carried out at much earlier stages to give a complete evaluation on the effects of self-gravity and then the back-reaction.

\section{Acknowledgments}

We thank Pisin Chen for his comments on the issue at the early stages of this work. Y.N. acknowledges enlightening discussions with Alexei Starobinsky. This work is supported by the National Natural Science Foundation of China (Grant Nos. 11021092, 10975003, and 11035003), and the National Fund for Fostering Talents of Basic Science (Grant Nos. J0630311, J0730316). It is also supported by the Education Foundation for Undergraduate Research and Hui-Chun Chin and Tsung-Dao Lee Chinese Undergraduate Research Endowment (Chun-Tsung Endowment) at Peking University.
[1] S. Dodelson, Modern Cosmology (Academic Press, San Diego, 2003).

[2] B. Carter and J.P. Luminet, Mon. Not. R. Astron. Soc. 212, 23 (1985).

[3] P. Wiggins and D. Lai, Astrophys. J. 532, 530 (2000) arXiv:astro-ph/9907365.

[4] D. J. Kaup, Phys. Rev. 172, 1331 (1968).

[5] R. Ruffini and S. Bonazzola, Phys. Rev. 187, 1767 (1969).

[6] G. Ingrosso and R. Ruffini, Nuovo Cimento B 101, 369 (1988).

[7] M. Laine and M. E. Shaposhnikov, Nucl. Phys. B 532, 376 (1998) arXiv:hep-ph/9804237.

[8] M. Colpi, S. L. Shapiro, and I. Wasserman, Phys. Rev. Lett. 57, 2485 (1986).

[9] P. Jetzer, Phys. Rept. 220, 163 (1992).
[10] J. Ho, S. j. Kim, and B. H. Lee, arXiv:gr-qc/9902040].

[11] S. W. Hawking, Phys. Rev. Lett. 26, 1344 (1971).

[12] B. J. Carr and S. W. Hawking, Mon. Not. Roy. Astron. Soc. 168, 399 (1974).

[13] N. Bilic and H. Nikolic, Nucl. Phys. B 590, 575 (2000) arXiv:gr-qc/0006065.

[14] R. Sharma, S. Karmakar, and S. Mukherjee, [arXiv:grqc/0812.3470].

[15] For a review, see H. J. de Vega, and N. G. Sanchez, Phys. Lett. B 490, 180 (2000) arXiv:hep-th/9903236.

[16] G. E. Uhlenbeck and L. Gropper, Phys. Rev. 41, 79 (1932).

[17] S. Weinberg, The first three minutes: a modern view of the origin of the universe (Basic Books, New York, 1993).

[18] S. F. Daniel, R. R. Caldwell, A. Cooray, and A. Melchiorri, Phys. Rev. D 77, 103513 (2008) [arXiv:astro- 
$\mathrm{ph} / 0802.1068]$.

[19] D. N. Spergel et al. [WMAP Collaboration], Astrophys. J. Suppl. 170, 377 (2007) arXiv:astro-ph/0603449.

[20] M. Peimbert, V. Luridiana, and A. Peimbert, Astrophys.
J. 666, 636 (2007) arXiv:astro-ph/0701580.

[21] G. Steigman, Ann. Rev. Nucl. Part. Sci. 57, 463 (2007) [arXiv:astro-ph/0712.1100]. 